\title{
Impact of electron-electron interactions induced by disorder at interfaces on spin-dependent tunneling in Co-Fe-B/MgO/Co-Fe-B magnetic tunnel junctions
}

\author{
J. Bernos, ${ }^{1}$ M. Hehn,,${ }^{1}$ F. Montaigne, ${ }^{1}$ C. Tiusan, ${ }^{1}$ D. Lacour, ${ }^{1}$ M. Alnot,${ }^{1}$ B. Negulescu, ${ }^{1}$ G. Lengaigne,${ }^{1}$ \\ E. Snoeck, ${ }^{2}$ and F. G. Aliev ${ }^{3}$ \\ ${ }^{1}$ Institut Jean Lamour, UMR CNRS 7198, Nancy Université, Bd. des Aiguillettes, BP 239, 54506 Vandoeuvre Les Nancy Cedex, France \\ ${ }^{2}$ CEMES, 29 rue Jeanne Marvig, F-31055 Toulouse, France \\ ${ }^{3}$ Departamento de Física de la Materia Condensada, Universidad Autónoma de Madrid, Cantoblanco 28049, Spain
}

(Received 4 August 2010; published 23 August 2010)

\begin{abstract}
In this study, we demonstrate that, beyond the standard magnon excitations, the electron-electron interactions in presence of disorder in $\mathrm{CoFeB}$ electrodes play a significant role on the zero-bias anomaly measured in $\mathrm{CoFeB} / \mathrm{MgO} / \mathrm{CoFeB}$ junctions. The low-temperature dependence of the tunneling conductance presents cusplike dip at low voltage varying as the square root of the bias voltage. The amplitude of this zero-bias anomaly decreases with the annealing temperature, indicating interface crystallization and related increase in the tunnel magnetoresistance.
\end{abstract}

DOI: 10.1103/PhysRevB.82.060405

Since the discovery of the magnetoresistance phenomenon in magnetic tunnel junctions (MTJs) at room temperature more than one decade ago, ${ }^{1}$ significant efforts have been devoted toward the understanding of the underlying physics. On the basis of theoretical predictions, ${ }^{2}$ the tunnel magnetoresistance (TMR) value has been brought to $600 \%$ (Ref. 3) using $\mathrm{CoFeB} / \mathrm{MgO} / \mathrm{CoFeB}$-sputtered MTJ. From a fundamental point of view, these epitaxial MgO-based MTJs represent a model system to address spin-dependent symmetry filtering effects occurring during the tunneling transfer of carriers. Within this context, a number of fundamental questions still remain to be clarified for a complete understanding of the fundamental physics. One of them relates to the socalled zero-bias anomaly (ZBA), a dip in the tunnel conductance around zero applied voltage, that links the variation in TMR to the applied voltage. This variation has to be controlled for many applications such as sensors, next generation of data storage devices or high-frequency oscillators.

The ZBA has been observed in numerous tunneling systems, and a wealth of mechanisms has been invoked to explain its origins. Large ZBAs have been extensively studied in nonmagnetic tunnel junctions including magnetic impurities or impurity layers placed either within one of the electrodes or within the insulating barrier. ${ }^{4}$ For magnetic impurities within a nonmagnetic electrode, the observed ZBAs were explained by considering the modification of the interfacial density of states by the impurities. Moreover, ZBAs have also been observed in magnetic tunnel junctions of different types: $\mathrm{Co} / \mathrm{AlOx} / \mathrm{CoFe},{ }^{5} \mathrm{LSMO} / \mathrm{STO} / \mathrm{LSMO},{ }^{6}$ and $\mathrm{CoFeB} / \mathrm{MgO} / \mathrm{CoFeB} .{ }^{7}$ In such systems, the role of magnons in inelastic tunneling has been highlighted both form theoretical and experimental point of view. ${ }^{5}$ Other studies concluded that Kondo effects in both magnetic and nonmagnetic tunnel junctions, with magnetic and nonmagnetic hosts, may be responsible for zero-bias anomalies. ${ }^{8,9}$ Finally, through the bias dependence of the tunnel characteristics, a metalinsulator transition in granular aluminum ${ }^{10}$ and amorphous $\mathrm{Ge}_{1-x} \mathrm{Au}_{x}$ films ${ }^{11}$ had been shown to be responsible for ZBA in nonmagnetic tunnel junctions. As far as magnetic tunnel junctions are concerned, direct observation of such effects was only briefly evoked in $\mathrm{CoFe} / \mathrm{AlOx} / \mathrm{CoFe}$ MTJ (Ref. 12)
PACS number(s): 75.76.+j, 73.20.Fz, 73.40.Gk, 73.40.Rw

even if the CoFe magnetic electrodes exhibit metallic properties and low resistivity.

In this Rapid Communication, we report the voltage and temperature dependence of the ZBA observed in the actual most studied $\mathrm{CoFeB} / \mathrm{MgO} / \mathrm{CoFeB}$ MTJs. We consider recent studies on magnetotransport properties of similar MTJ systems and review the possible origins for the ZBA. We argue that an hitherto neglected contribution of electronlocalization phenomena, occurring in the partially disordered $\mathrm{CoFeB}$ electrodes, accounts for the ZBA seen in the parallel magnetizations configuration at applied voltages less than 50 $\mathrm{mV}$ and measure temperatures less than $50 \mathrm{~K}$. Since amorphous $\mathrm{CoFeB}$ films are known to exhibit a metal-insulator transition, ${ }^{13}$ we analyze the behavior of the conductancevoltage characteristics within the context of early studies of disordered systems with a metal-insulator transition and show that the measurement of the ZBA is an indirect measure of the interface crystallization. Furthermore, $\mathrm{CoFeB} /$ $\mathrm{MgO} / \mathrm{CoFeB}$ appears to be a unique system to follow the crystallization of the electrodes and the related evolution of ZBA, correlated with the TMR. Indeed while in other studies different samples are required to change the amorphicity and the related band structure of the electrodes, here one sample submitted to an increasing annealing temperature allows to track the evolution of the band structure with a progressive electrode crystallization.

The MTJs presented in this study were deposited on glass substrates by sputtering and have the following structure: $\mathrm{Ta}(5) / \mathrm{Co}(10) / \mathrm{IrMn}(7.5) / \mathrm{CoFeB}(4) / \mathrm{MgO}\left(t_{\mathrm{MgO}}\right) /$ $\mathrm{CoFeB}(5) / \mathrm{Ta}(5) / \mathrm{Pt}(3)$. The thickness of each layer is given in nanometers; the $\mathrm{MgO}$ barrier thickness $t_{\mathrm{MgO}}$ was varied from 2 to $3 \mathrm{~nm}$. The $\mathrm{CoFeB}$ was rf deposited from a $\mathrm{Co}_{40} \mathrm{Fe}_{40} \mathrm{~B}_{20}$ alloy target. A $1 \mathrm{~h}$ postdeposition annealing process was done in a high-vacuum chamber varying the annealing temperature, $T_{\text {ann }}$, between 200 and $350^{\circ} \mathrm{C}$, under an applied magnetic field of 200 Oe. Figure 1 summarizes measurements made on our MTJs. Junction resistance vs applied field, measured at $+10 \mathrm{mV}$, shows decoupled magnetic layers with an increase in TMR at low temperatures [Fig. 1(a)]. Figure 1(b) presents the TMR vs $t_{\mathrm{MgO}}$ for three different values of $T_{\mathrm{ann}}$. For $T_{\mathrm{ann}}=200{ }^{\circ} \mathrm{C}$, the TMR first increases and 

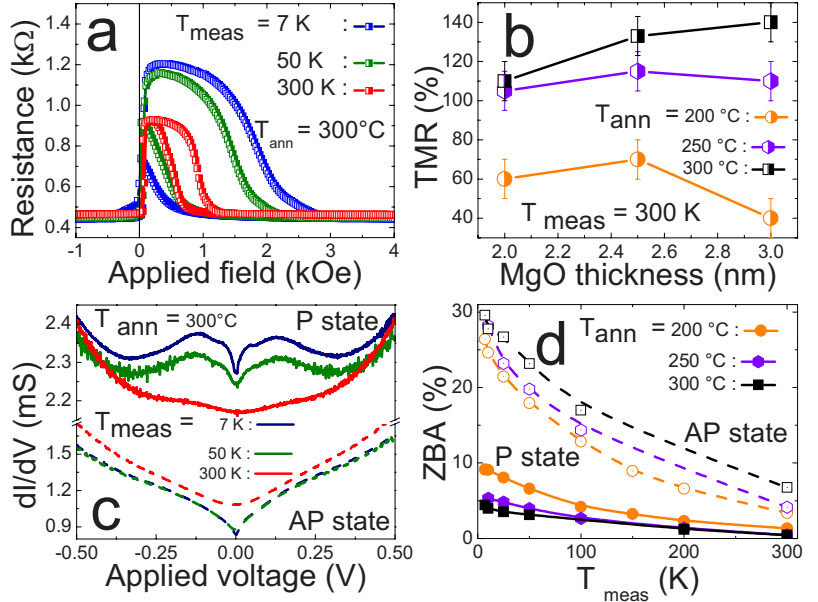

FIG. 1. (Color online) (a) Resistance vs applied field measured at different temperatures $\left(T_{\text {meas }}\right)$ on a $\operatorname{MTJ}\left(t_{\mathrm{MgO}}=2 \mathrm{~nm}, T_{\text {ann }}\right.$ $=300{ }^{\circ} \mathrm{C}$ ); (b) TMR vs $t_{\mathrm{MgO}}$ measured on MTJ at $300 \mathrm{~K}$ for three different values of $T_{\mathrm{ann}}$. Error bars were evaluated from the measure on more than ten different junctions. (c) Differential conductances measured at different values of $T_{\text {meas }}$ on a MTJ $\left(t_{\mathrm{MgO}}\right.$ $=2 \mathrm{~nm}, T_{\mathrm{ann}}=300{ }^{\circ} \mathrm{C}$ ) in the $\mathrm{P}($ continuous lines) and $\mathrm{AP}$ (dashed lines) states. (d) ZBA vs $T_{\text {meas }}$ measured on $\operatorname{MTJ}\left(t_{\mathrm{MgO}}=2 \mathrm{~nm}\right)$ measured in the $\mathrm{P}$ (continuous lines) and AP (dashed lines) states for three different values of $T_{\text {ann }}$.

then decreases when $t_{\mathrm{MgO}}$ increases. This is not the case for $T_{\text {ann }}=300{ }^{\circ} \mathrm{C}$ : the TMR is always increasing in the studied range of $\mathrm{MgO}$ thicknesses. Moreover at constant thickness, the TMR increases when $T_{\text {ann }}$ increases. All these described features are consistent with a progressive crystallization of the tunneling system including $\mathrm{MgO}$ barrier and $\mathrm{CoFeB}$ interfaces. Indeed the TMR decrease observed for $T_{\text {ann }}$ $<300{ }^{\circ} \mathrm{C}$ is typical of amorphous barriers and this general tendency can be explained in a parabolic band framework. ${ }^{14}$ On the contrary, for $T_{\text {ann }}=300{ }^{\circ} \mathrm{C}$, the TMR continuous increase is consistent with a symmetry-dependent filtering effect of the electronic Bloch wave functions into the $\mathrm{MgO}$ barrier. $^{2}$ The observation of this effect reveals a crystallization of the tunneling system. The progressive crystallization with increasing $T_{\text {ann }}$ is confirmed by the following analysis of the dynamical conductances $[G(V)=d I / d V(V)]$. Figure 1(c) displays the $G(V)$ 's recorded for a MTJ with a $2 \mathrm{~nm}$ thick $\mathrm{MgO}$ barrier, in the parallel (P) and antiparallel (AP) states of magnetizations, at various temperatures $\left(T_{\text {meas }}\right)$, and after annealing at $T_{\mathrm{ann}}=300{ }^{\circ} \mathrm{C}$. A double minima at $375 \mathrm{mV}$, attributed to the contribution of the $\Delta_{5}$ majority electron channel or $\Delta_{1}$ minority electron channel to the tunnel process is present at all temperatures, especially at low temperatures. This is a symmetry filtering effect resulting from the increasing crystalline quality of the $\mathrm{CoFeB} / \mathrm{MgO}$ interface induced by the thermal anneal. ${ }^{15}$ However, at temperatures less then $50 \mathrm{~K}$ and applied voltage less than $50 \mathrm{mV}$, a ZBA materialized by a cusplike dip in the tunneling conductance at low voltage is visible in both $\mathrm{P}$ and AP states. This ZBA was not observed in fully epitaxial tunnel junctions. For further analysis of the ZBA, we quantify it in both the $\mathrm{P}$ and AP states as: $\mathrm{ZBA}=100[G(100 \mathrm{mV})-G(0 \mathrm{mV})] / G(0 \mathrm{mV})$. The ZBA evolution with temperature $\left(T_{\text {meas }}\right)$ and with $T_{\text {ann }}$ is

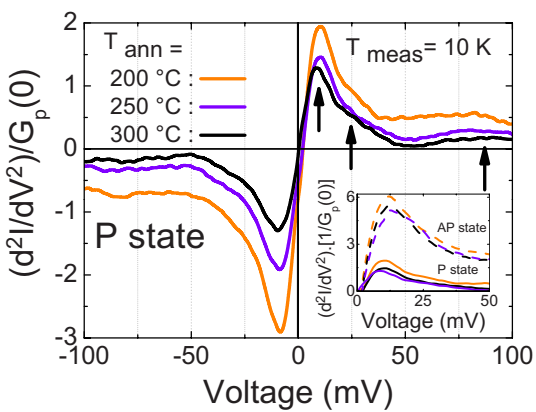

FIG. 2. (Color online) Normalized $d^{2} I / d V^{2}$ vs voltage, measured at $10 \mathrm{~K}$ on a MTJ having a 2-nm-thick MgO barrier. Inset: zoom of the normalized $d^{2} I / d V^{2}$ vs voltage for applied voltage between 0 and $50 \mathrm{mV}$ measured at $10 \mathrm{~K}$ on the same junction for the $\mathrm{P}$ and $\mathrm{AP}$ states.

plotted in Fig. 1(d). The ZBA in the P state is significantly lower than in the AP state. In both cases, the ZBA decreases as $T_{\text {meas }}$ increases. However, the evolution of the ZBA with increasing $T_{\text {ann }}$ is completely opposite in the P and AP states. While the ZBA decreases with an increase in $T_{\text {ann }}$ in $\mathrm{P}$ state, it increases in the AP state. This suggests that different tunneling channels and related transport mechanisms are involved in the two magnetic states. In the $\mathrm{P}$ configuration, the ZBA evolution appears to be inversely correlated with that of the TMR versus annealing temperature. Finally, the ZBA decreases by a factor of two when the $\mathrm{MgO}$ thickness is brought from 2 to $3 \mathrm{~nm}$ (not shown).

In order to identify the fundamental interactions responsible for the ZBA, $d^{2} I / d V^{2}(V)$ inelastic electron-tunneling spectra (IETS) have been numerically computed from the $d I / d V(V)$ data at $10 \mathrm{~K}$, for different $T_{\text {ann }}$. As can be seen in Fig. 2, several peaks are present in the region $-100 \mathrm{mV} / 100 \mathrm{mV}$ in which the ZBA occurs. A first peak appears at very low bias $(10 \mathrm{mV})$; a second, wide shoulderlike peak is present around $35 \mathrm{mV}$; and a third, wider and smaller peak is visible around $80 \mathrm{mV}$. The latter is usually considered to be the signature of $\mathrm{MgO}$ phonon mode. ${ }^{16}$ The origin of the two first peaks is not clear, and there are some disagreements about their interpretation in the literature. ${ }^{16-20}$ The first is usually attributed to magnetic impurity scattering and the second to magnon excitation. ${ }^{17-20}$ Others similar studies, ${ }^{20}$ show no second peak; the remaining peak being attributed to magnon excitations. As the two electrodes are ferromagnetic, the presence of a peak in IETS associated to magnon excitations is expected in the range of considered energy. More surprising is the presence of an additional peak. As this last one has been associated to the presence of impurities in the tunneling barrier we will first comment on the quality of our tunnel barrier. From a spin-dependent transport point of view, as reported by Lu et al. ${ }^{21}$ electronic states localized in the tunneling barrier of such systems imply a drop of TMR with an increase in $t_{\mathrm{MgO}}$. Indeed, increasing the barrier thickness would lead to more localized defects in the barrier and an enhancement of the chain-hopping contribution to junction conductance. In our samples, the increase in the barrier thickness leads to a decrease in the ZBA and an increase in the TMR [see Fig. 1(b)]. So, from a spindependent transport point of view, we do not have evidence 

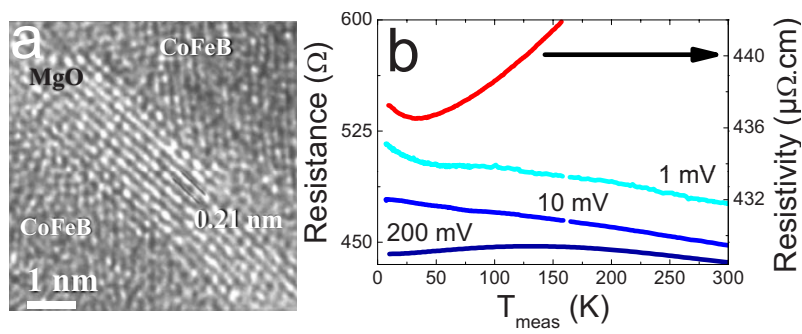

FIG. 3. (Color online) (Color online): (a) TEM image of a MTJ $\left(t_{\mathrm{MgO}}=2 \mathrm{~nm}\right)$ after a thermal anneal at $300{ }^{\circ} \mathrm{C}$. (b) Resistivity vs $T_{\text {meas }}$ for a $5 \mathrm{~nm}$ thick not annealed CoFeB rod (red). Resistance vs $T_{\text {meas }}$ measured on a MTJ $\left(t_{\mathrm{MgO}}=2 \mathrm{~nm}\right)$ in the P state at 1,10 , and $200 \mathrm{mV}$ after annealing at $200{ }^{\circ} \mathrm{C}$.

of impurities presence in our tunnel barrier. This analysis based on transport measurements is supported by x-ray photoemission spectroscopy (XPS) analysis made on our samples in order to determine their chemical composition. We focused the analysis on the two elements $\mathrm{Mn}$ and B, whose propensity for thermally activated diffusion in such structures is well known. We observed that both the CoFeB electrodes close to the interface and the barrier are $\mathrm{Mn}$ free, even after annealing at $300{ }^{\circ} \mathrm{C}$. Thus, within the sensitivity of our analysis, we can rule out the presence of magnetic impurities into the $\mathrm{MgO}$ layer. Concerning localized nonmagnetic impurities within the barrier (e.g., boron), the XPS $\mathrm{B} 1 s$ spectra indicate that the $\mathrm{B}$ atoms do not diffuse in large quantities into the $\mathrm{MgO}$ and that they are uniformly distributed inside the barrier. Furthermore, the concentration of boron does not evolve for annealing temperatures between 200 and $300{ }^{\circ} \mathrm{C}$. Therefore both the variation in ZBA with $T_{\text {ann }}$ and the presence of an additional peak in IETS cannot be ascribed to transport assisted by impurities or defaults. Finally since the amplitudes of the two peaks depend on the magnetizations configurations in our study (see inset of Fig. 2 ), none of these should be attributed to magnetic-impurity scattering.

The structural analysis made by $\mathrm{x}$-ray characterization (not shown) and by high-resolution transmission electron microscopy (HRTEM) [Fig. 3(a)] shows the existence of a dominant amorphous phase in the CoFeB electrodes. The electrical characterization of a $10-\mu \mathrm{m}$-long, $10-\mu \mathrm{m}$-wide, and 5-nm-thick CoFeB rod grown under the same conditions of our electrodes is reported in Fig. 3(b) (red curve). The resistivity vs temperature presents a minimum at around 40 $\mathrm{K}$. This behavior is characteristic of a metal-insulator transition exhibited by amorphous metallic films. ${ }^{22}$ This minimum vanishes after a thermal anneal at $370{ }^{\circ} \mathrm{C}$. Our hypothesis of electron-electron interactions in the $\mathrm{CoFeB}$ electrodes as origin of the additional peak in IETS stems from the direct analysis of this peculiar low-temperature variation in the $\mathrm{CoFeB}$ resistance. The transition of the amorphous junction's electrode should have a direct impact on the tunneling conductance as it probes directly any electronic-structure modifications in the $\mathrm{CoFeB}$ layer. And indeed the weaklocalization effect in the $\mathrm{CoFeB}$ film has a clear signature in the variation in the junction resistance vs $T_{\text {meas }}$ [blue curves in Fig. 3(b)]. One can see that in the ZBA regime (small bias voltage $1-10 \mathrm{mV}$ ), a net increase in the junction resistance

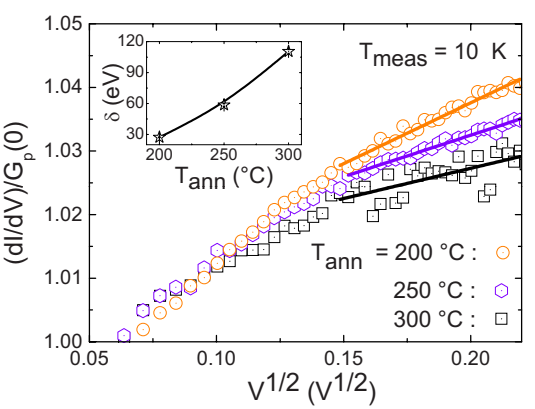

FIG. 4. (Color online) Normalized $d I / d V$ vs $V^{1 / 2}$ measured at 10 $\mathrm{K}$ for different $T_{\mathrm{ann}}$ in the $\mathrm{P}$ state. Straight lines are fits. Inset: values of $\delta$ extracted from fits.

appears below $50 \mathrm{~K} .^{23}$ This correlates with the transition observed around $40 \mathrm{~K}$ for the single CoFeB rod. In contrast, for larger bias voltages (e.g., $200 \mathrm{mV}$ ), the variation in the resistance vs $T_{\text {meas }}$ in the $\mathrm{P}$ state corresponds to a standard behavior observed also in single-crystal MTJ devices. ${ }^{24}$ This standard behavior has been explained in terms of misalignment of magnetic moments in the electrodes due to thermal excitations. Therefore, the increase in junction resistance measured at low voltage $(1 \mathrm{mV})$ and at low temperatures can be related to a density-of-state (DOS) reduction around the Fermi level in the amorphous CoFeB. Indeed, while a disordered system is transiting from a metallic to an insulating state, the electronic mean free path becomes comparable to the Fermi wavelength and the electron-electron interaction leads to a symmetric DOS depletion around the Fermi level. ${ }^{25}$ In the limit of strong scattering and using a scaling model that includes localization, correlation, and screening, the predicted density-of-states variation is given by ${ }^{11,25}$ $N(E)=N(0)(1+\sqrt{E / \delta})$, where the correlation gap $\delta$ is a measure of the screening length. This additional shape of the band structure should lead to a contribution proportional to $V^{1 / 2}$ at the total conductance, in addition to the magnons one. Then, increasing the disorder leads to a divergence of the screening length and $\delta$ should converge to zero.

To check this hypothesis we have plotted $G(V)$ as a function of $V^{1 / 2}$ in Fig. 4. We clearly observed two regimes, one between 0 and $20 \mathrm{mV}$ where both magnons and electronselectrons interactions are contributing to the $G(V)$ variation and one between 20 and $50 \mathrm{mV}$, where the magnon contribution is saturated. We have fitted $G(V)$ curves for applied voltages between 23 and $50 \mathrm{mV}$ with a $V^{1 / 2}$ law to extract the so-called pseudocorrelation-gap parameter $\delta$. This analysis as a function of the annealing temperature makes sense only in the P state of junction magnetizations where our measurements probe a dominant majority-spin conduction channel and its related density of states. In the AP state, the minority DOS is probed and evolution of pseudocorrelation gap as a function of annealing temperature becomes impossible due to an additional electronic-structure effect: with crystallization of $\mathrm{CoFeB}$, a pseudogap specific to the bcc structures opens up around the Fermi level in the minority DOS.

The results of our quantitative analysis are given in the inset of Fig. 4. First of all, these values of the correlation gap in $\mathrm{CoFeB}$ are consistent with those reported for $\mathrm{Fe}_{80} \mathrm{Sn}_{20}$ $(\delta=20 \mathrm{eV})$ (Ref. 26) and $\mathrm{Fe}_{80} \mathrm{~B}_{20}(\delta=256 \mathrm{eV})$ (Ref. 27) in 
bulk materials. We observe that, increasing $T_{\text {ann }}$ leads to an increase in $\delta$, directly related to the reduction in the ZBA and the increase in the TMR. This increase is a sign of the decrease in disorder at $\mathrm{CoFeB} / \mathrm{MgO}$ interface due to the crystallization of the CoFeB. However, in our samples, the crystallization is not perfect (see HRTEM analysis) and the ZBA persists even postannealing. This would also explain the reduced amplitude of TMR ratios measured in our junctions $\left(150 \%\right.$ at $\left.300{ }^{\circ} \mathrm{C}\right)$, compared to the best reported value $(600 \%)$. At this point, it is still difficult to mark out the exact contributions of the increasingly efficient filtering effects and of the gradual disappearance of the localization effects.

In conclusion, at low temperatures, we observe a cusplike dip in the tunneling. We found that the conductance contains a contribution varying as the square root of the bias voltage, which, in light of the temperature dependence displayed by the resistivity of $\mathrm{CoFeB}$ films, suggests strongly the presence of electron-electron interactions close to the interface between the partially amorphous ferromagnetic electrodes and the $\mathrm{MgO}$ barrier. We interpret the increase in the pseudocorrelation-gap parameter in the $\mathrm{P}$ configuration with increasing $T_{\text {ann }}$ as a sign of the disorder decrease at the $\mathrm{CoFeB} / \mathrm{MgO}$ interface. Thus, the $\mathrm{CoFeB}$ crystallization leads to an increase in the TMR signal. Additionally, in the $\mathrm{P}$ configuration, a direct link between ZBA, the increase in TMR, and the appearance of specific symmetry-dependent features in the conductance have been highlighted.

The authors would like to thank D. Malterre, Ph. Mangin, and F. Gautier for fruitful discussions, S. Suire for technical assistance, and S. Girod for help with some measurements. This work was partially supported by ANR contracts CTS Lor-Al, Spinchat, SpinPress, and Spanish-French bilateral under Project No. FR2009-0010. One of the authors (E.S.) acknowledges financial support from the CNRS and CEA METSA network. F.G.A. acknowledges support by Spanish MICINN (MAT2009-10139, CSD2007-00010) and CAM (P2009/MAT-1726).
${ }^{1}$ J. S. Moodera, L. R. Kinder, T. M. Wong, and R. Meservey, Phys. Rev. Lett. 74, 3273 (1995).

${ }^{2}$ W. H. Butler, X.-G. Zhang, T. C. Schulthess, and J. M. MacLaren, Phys. Rev. B 63, 054416 (2001); J. Mathon and A. Umerski, ibid. 63, 220403 (2001).

${ }^{3}$ S. S. P. Parkin, C. Kaiser, A. Panchula, P. M. Rice, B. Hughes, M. Samant, and S.-H. Yang, Nature Mater. 3, 862 (2004); S. Yuasa, T. Nagahama, A. Fukushima, Y. Suzuki, and K. Ando, ibid. 3, 868 (2004); S. Ikeda, J. Hayakawa, Y. Ashizawa, Y. M. Lee, K. Miura, H. Hasegawa, M. Tsunoda, F. Matsukura, and H. Ohno, Appl. Phys. Lett. 93, 082508 (2008).

${ }^{4}$ E. L. Wolf, Principles of Electron Tunneling Spectroscopy (Oxford University Press, London, 1985), Chap. 8, for an extensive review.

${ }^{5}$ S. Zhang, P. M. Levy, A. C. Marley, and S. S. P. Parkin, Phys. Rev. Lett. 79, 3744 (1997).

${ }^{6}$ J. O’Donnell, A. E. Andrus, S. Oh, and J. N. Eckstein, Appl. Phys. Lett. 76, 1914 (2000); J. Z. Sun, D. W. Abraham, K. Roche, and S. S. P. Parkin, ibid. 73, 1008 (1998).

${ }^{7}$ R. Matsumoto, S. Nishioka, M. Mizuguchi, M. Shiraishi, H. Maehara, K. Tsunekawa, D. D. Djayaprawira, N. Watanabe, Y. Otani, T. Nagahama, A. Fukushima, H. Kubota, S. Yuasa, and Y. Suzuki, Solid State Commun. 143, 574 (2007); D. V. Dimitrov, Z. Gao, X. Wang, W. Jung, X. Lou, and O. Heinonen, J. Appl. Phys. 105, 113905 (2009); S. Ikegawa, H. Aikawa, T. Ueda, M. Nagamine, N. Shimomura, M. Yoshikawa, K. Hosotani, and H. Yoda, ibid. 101, 09B504 (2007).

${ }^{8}$ K. I. Lee, S. J. Joo, J. H. Lee, K. Rhie, T.-S. Kim, W. Y. Lee, K. H. Shin, B. C. Lee, P. LeClair, J.-S. Lee, and J.-H. Park, Phys. Rev. Lett. 98, 107202 (2007).

${ }^{9}$ P. LeClair, J. T. Kohlhepp, H. J. M. Swagten, and W. J. M. de Jonge, Phys. Rev. Lett. 86, 1066 (2001).

${ }^{10}$ R. C. Dynes and J. P. Garno, Phys. Rev. Lett. 46, 137 (1981).

${ }^{11}$ W. L. McMillan and J. Mochel, Phys. Rev. Lett. 46, 556 (1981); W. L. McMillan, Phys. Rev. B 24, 2739 (1981).

${ }^{12}$ E. R. Nowak, P. Spradling, M. B. Weissman, and S. S. P. Parkin, Thin Solid Films 377-378, 699 (2000).

${ }^{13}$ S. U. Jen, Y. D. Yao, Y. T. Chen, J. M. Wu, C. C. Lee, T. L. Tsai, and Y. C. Chang, J. Appl. Phys. 99, 053701 (2006).

${ }^{14}$ F. Montaigne, M. Hehn, and A. Schuhl, Phys. Rev. B 64, 144402 (2001).

${ }^{15}$ C. Tiusan, M. Hehn, F. Montaigne, F. Greullet, S. Andrieu, and A. Schuhl, J. Phys.: Condens. Matter 19, 165201 (2007).

${ }^{16}$ G.-X. Miao, K. B. Chetry, A. Gupta, W. H. Butler, K. Tsunekawa, D. Djayaprawira, and G. Xiao, J. Appl. Phys. 99, $08 \mathrm{~T} 305$ (2006).

${ }^{17}$ K. Tamanoi, M. Sato, M. Oogane, Y. Ando, T. Tanaka, Y. Uehara, and T. Uzumaki, J. Magn. Magn. Mater. 320, 2959 (2008).

${ }^{18}$ R. Matsumoto, Y. Hamada, M. Mizuguchi, M. Shiraishi, H. Maehara, K. Tsunekawa, D. D. Djayaprawira, N. Watanabe, Y. Kurosaki, T. Nagahama, A. Fukushima, H. Kubota, S. Yuasa, and Y. Suzuki, Solid State Commun. 136, 611 (2005).

${ }^{19}$ S. Nishioka, Y. V. Hamada, R. Matsumoto, M. Mizuguchi, M. Shiraishi, A. Fukushima, H. Kubota, T. Nagahama, S. Yuasa, H. Maehara, Y. Nagamine, K. Tsunekawa, D. D. Djayaprawira, N. Watanabe, and Y. Suzuki, J. Magn. Magn. Mater. 310, e649 (2007).

${ }^{20}$ V. Drewello, M. Schafers, O. Schebaum, A. A. Khan, J. Munchenberger, J. Schmalhorst, G. Reiss, and A. Thomas, Phys. Rev. B 79, 174417 (2009).

${ }^{21}$ Y. Lu, M. Tran, H. Jaffres, P. Seneor, C. Deranlot, F. Petroff, J.-M. George, B. Lepine, S. Ababou, and G. Jezequel, Phys. Rev. Lett. 102, 176801 (2009).

${ }^{22}$ V. M. Agranovich and A. A. Maradudin, Modern Problems in Condensed Matter Sciences (North-Holland, Amsterdam, 1985), Vol. 10.

${ }^{23}$ This net increase is reduced after thermal anneal at higher temperature. The same trend is observed for thicker $\mathrm{MgO}$ barriers.

${ }^{24}$ Q. L. Ma, S. G. Wang, J. Zhang, Y. Wang, R. C. C. Ward, C. Wang, A. Kohn, X.-G. Zhang, and X. F. Han, Appl. Phys. Lett. 95, 052506 (2009).

${ }^{25}$ B. L. Altshuler and A. G. Aronov, Solid State Commun. 30, 115 (1979).

${ }^{26}$ G. Xiao and C. L. Chien, Phys. Rev. B 34, 8430 (1986).

${ }^{27}$ R. W. Cochrane and J. O. Strom-Olsen, Phys. Rev. B 29, 1088 (1984). 